\title{
CONNECTIVITY OF RANDOM GEOMETRIC GRAPHS RELATED TO MINIMAL SPANNING FORESTS
}

\author{
C. $\mathrm{HIRSCH},{ }^{* * *}$ \\ D. NEUHÄUSER * AND \\ V. SCHMIDT, ${ }^{*}$ Ulm University
}

\begin{abstract}
The almost-sure connectivity of the Euclidean minimal spanning forest $\operatorname{MSF}(X)$ on a homogeneous Poisson point process $X \subset \mathbb{R}^{d}$ is an open problem for dimension $d>2$. We introduce a descending family of graphs $\left(G_{n}\right)_{n \geq 2}$ that can be seen as approximations to the MSF in the sense that $\operatorname{MSF}(X)=\bigcap_{n=2}^{\infty} G_{n}(X)$. For $n=2$, one recovers the relative neighborhood graph or, in other words, the $\beta$-skeleton with $\beta=2$. We show that almost-sure connectivity of $G_{n}(X)$ holds for all $n \geq 2$, all dimensions $d \geq 2$, and also point processes $X$ more general than the homogeneous Poisson point process. In particular, we show that almost-sure connectivity holds if certain continuum percolation thresholds are strictly positive or, more generally, if almost surely $X$ does not admit generalized descending chains.
\end{abstract}

Keywords: Minimal spanning forest; descending chain; $\beta$-skeleton; point process; continuum percolation

2010 Mathematics Subject Classification: Primary 60D05

Secondary $05 \mathrm{C} 80 ; 05 \mathrm{C} 10 ; 82 \mathrm{~B} 43$

\section{Introduction}

Aldous and Steele [4] introduced the notion of a Euclidean minimal spanning forest $\operatorname{MSF}(\varphi)$ on a locally finite subset $\varphi \subset \mathbb{R}^{d}, d \geq 2$. This is a geometric graph with vertex set $\varphi$ and edge set determined by the creek-crossing property. That is, $x, y \in \varphi$ are connected by an edge in $\operatorname{MSF}(\varphi)$ if there do not exist an integer $m$ and a finite sequence of vertices $x_{0}=x, \ldots, x_{m}=y, x_{i} \in \varphi$ for all $i=0, \ldots, m$ satisfying $\left|x_{i}-x_{i+1}\right|<|x-y|$ for all $i=0, \ldots, m-1$. Aldous and Steele [4] furthermore conjectured that the $\operatorname{MSF}(X)$ is almost surely (a.s.) connected for $X \subset \mathbb{R}^{d}$ a homogeneous Poisson point process (PPP). In [6] this conjecture was proven for dimension $d=2$. However, now there is a strong belief that almost-sure connectivity does not hold as soon as the dimension $d$ is large enough (see, e.g. [5, Section 6.3]). The counterparts of the MSF for lattice graphs are studied in [15] and [19], partly due to their intimate connection to percolation theory. Connectivity and related properties of the Euclidean MSF are discussed in [6] and also in [2] (in the general context of unimodular random networks).

In this paper, for each integer $n \geq 2$, we consider a geometric graph, which is defined by a truncated version of the creek-crossing property and called a creek-crossing graph of order $n$. More precisely, for $\varphi \subset \mathbb{R}^{d}$ locally finite, let $G_{n}(\varphi)$ be a graph with vertex set $\varphi$ where two vertices $x, y \in \varphi$ are connected by an edge in $G_{n}(\varphi)$ if there do not exist an integer $m \leq n$ and

Received 3 November 2011; revision received 3 May 2012.

* Postal address: Institute of Stochastics, Ulm University, 89069 Ulm, Germany.

** Email address: christian.hirsch@uni-ulm.de 
vertices $x_{0}=x, x_{1}, \ldots, x_{m}=y \in \varphi$ such that $\left|x_{i}-x_{i+1}\right|<|x-y|$ for all $i \in\{0, \ldots, m-1\}$. Observe that

$$
G_{2}(\varphi) \supset G_{3}(\varphi) \supset \cdots \supset \bigcap_{i=2}^{\infty} G_{i}(\varphi)=\operatorname{MSF}(\varphi),
$$

which explains why we consider these graphs as approximations to the MSF. Furthermore, the graph $G_{2}(\varphi)$ is also known as the relative neighborhood graph (or $\beta$-skeleton with $\beta=2$ ) and was originally introduced in [20] in the context of computational geometry. Limit theorems for certain functionals on these graphs are considered in [17] and also [18]. In particular, the inclusions

$$
\operatorname{Del}(\varphi) \supset G(1, \varphi) \supset G(\beta, \varphi) \supset G(2, \varphi)=G_{2}(\varphi)
$$

hold, with $\operatorname{Del}(\varphi)$ and $G(\beta, \varphi)$ denoting the Delaunay triangulation and $\beta$-skeleton on $\varphi$, respectively, $1 \leq \beta \leq 2$.

The goal of this paper is to give criteria for point processes $X \subset \mathbb{R}^{d}$ ensuring the almost-sure connectivity of $G_{n}(X)$. Although the conjecture on the almost-sure connectivity of $\operatorname{MSF}(X)$ for $X \subset \mathbb{R}^{d}$ a homogeneous PPP is still open for $d>2$ (and may well depend on the dimension $d$ ), the connectivity of $G_{n}(X)$ is far more accessible: our criteria work in all dimensions and for point processes more general than the homogeneous PPP. Thus, in a certain sense, we provide conditions that the $\operatorname{MSF}(X)$ is 'almost' connected. From this perspective, they can be seen as a Euclidean counterpart of Theorem 1.3 of [15].

Note that relative neighborhood graphs (or $\beta$-skeletons) on point processes in $\mathbb{R}^{2}$ could potentially be considered as stochastic models for road systems. Also, in this context connectivity is a desirable property; see [1], [3], and [16]. An application of creek-crossing graphs in $\mathbb{R}^{3}$ has recently been considered in [9] in order to investigate the connectivity of nanoparticle systems in advanced functional materials.

We finally remark that, for $d=2$, it would be interesting to know under which conditions on $X$ the cells of $G_{n}(X)$ are a.s. bounded. This will be the subject of a forthcoming paper. For such stationary point processes, one can define a center function on the cells of $G_{n}(X)$ by using for instance the center of gravity for each cell. This allows us to consider $G_{n}(X)$ as a stationary partition of the Euclidean plane in the sense of [12].

\section{Connectivity of relative neighborhood graphs}

In order to provide the reader with a gentle introduction, we start by discussing the special case $n=2$ in detail.

\subsection{Descending chains}

Let us first recall the definition of a descending chain with respect to a locally finite subset of $\mathbb{R}^{d}$; see [8] and [11].

Definition 2.1. Let $\varphi \subset \mathbb{R}^{d}$ be a locally finite set. We say that $\varphi$ has a descending chain if there exists an infinite sequence $x_{1}, x_{2}, \ldots \in \varphi$ satisfying $\left|x_{i+1}-x_{i+2}\right|<\left|x_{i}-x_{i+1}\right|$ for all $i \geq 1$.

It is easy to see that each locally finite set $\varphi \subset \mathbb{R}^{d}$, which possesses a descending chain, also has a descending chain consisting of pairwise distinct elements; see Lemma 3.1 below for a generalized version of this statement. Moreover, the absence of descending chains in $\varphi$ implies connectivity of the relative neighborhood graph $G_{2}(\varphi)$. 
Theorem 2.1. Let $\varphi \subset \mathbb{R}^{d}$ be locally finite. If $\varphi$ has no descending chain then $G_{2}(\varphi)$ is connected.

Proof. In order to show the assertion of the theorem, we can actually use a similar construction as in [1]. First observe that $G_{2}(\varphi)$ is always connected if $\varphi$ is finite. Suppose now that $\varphi$ is an infinite set such that $G_{2}(\varphi)$ is not connected, where we write $x \neq y$ if $x, y \in \varphi$ do not belong to the same connected component of $G_{2}(\varphi)$; otherwise, we write $x \sim y$. Then, an infinite sequence $x_{1}, x_{2}, \ldots \in \varphi$ forming a descending chain can be constructed as follows. Choose an arbitrary $x_{1} \in \varphi$, and put

$$
x_{2}=\operatorname{argmin}_{y \in \varphi}\left(\left|x_{1}-y\right|: x_{1} \not y\right) .
$$

Now suppose that, for some $k \geq 2$, a sequence $x_{1}, \ldots, x_{k} \in \varphi$ is given such that

$$
x_{i}=\operatorname{argmin}_{y \in \varphi}\left(\left|x_{i-1}-y\right|: x_{i-1} \nsim y\right)
$$

holds for all $2 \leq i \leq k$ and such that $\left|x_{1}-x_{2}\right|>\cdots>\left|x_{k-1}-x_{k}\right|$. Then put

$$
x_{k+1}=\operatorname{argmin}_{y \in \varphi}\left(\left|x_{k}-y\right|: x_{k} \nsim y\right) .
$$

It is clear that $\left|x_{k-1}-x_{k}\right| \geq\left|x_{k}-x_{k+1}\right|$. Suppose that $\left|x_{k-1}-x_{k}\right|=\left|x_{k}-x_{k+1}\right|$. Then, as $x_{k-1} \nsucc x_{k}$, there exist $z \in \varphi$ such that

$$
\max \left\{\left|x_{k-1}-z\right|,\left|x_{k}-z\right|\right\}<\left|x_{k-1}-x_{k}\right|=\left|x_{k}-x_{k+1}\right| \text {. }
$$

This implies that $x_{k-1} \sim z \sim x_{k}$, leading to a contradiction. Thus, $\left|x_{k-1}-x_{k}\right|>\left|x_{k}-x_{k+1}\right|$, which proves the theorem.

The following result is an immediate consequence of Theorem 2.1.

Corollary 2.1. Let $X \subset \mathbb{R}^{d}$ be an arbitrary point process. If $X$ has no descending chain with probability 1 then $G_{2}(X)$ is a.s. connected.

\subsection{Annulus continuum percolation threshold}

In this subsection we discuss a connectivity condition for relative neighborhood graphs $G_{2}(X)$ induced by random point processes $X$, which is stronger but easier to verify than the condition formulated in Corollary 2.1. In connection with this, the following notion of an annulus continuum percolation threshold is useful.

Definition 2.2. Let $\varphi \subset \mathbb{R}^{d}$ be a locally finite set, and let $r \geq 0$ and $\varepsilon>0$. Consider the graph $G(\varphi, r, \varepsilon)$ with vertex set $\varphi$ and the following edge set. Assume that $x, y \in \varphi$ are connected by an edge if and only if $r<|x-y|<r+\varepsilon$. Furthermore, for $r \geq 0$ and a point process $X \subset \mathbb{R}^{d}$, we define the annulus continuum percolation threshold $\varepsilon_{c}(X, r)=\inf \{\varepsilon>$ $0: \mathbb{P}(G(X, r, \varepsilon)$ percolates $)>0\}$, where we say that a graph percolates if it contains an infinite self-avoiding path.

It is easy to see that $G(\varphi, r, \varepsilon)$ percolates for $r=\lim _{n \rightarrow \infty}\left|x_{n+1}-x_{n}\right|$ and all $\varepsilon>0$ if $x_{1}, x_{2}, \ldots$ is a descending chain of $\varphi$. Moreover, a probabilistic counterpart of this elementary statement is true, where we use the following auxiliary result.

Lemma 2.1. Let $f:[0, \infty) \rightarrow[0, \infty)$ be a function which satisfies $f(x)>x$ for all $x \in$ $[0, \infty)$. Then there exists a countable set $\mathbb{C} \subset \mathbb{R}$ such that $[0, \infty)=\bigcup_{q \in \mathbb{C} \cap[0, \infty)}[q, f(q))$. 
Proof. Let $\mathbb{Q} \subset \mathbb{R}$ denote the set of rational numbers. For each $q \in \mathbb{Q} \cap[0, \infty)$, define $\ell(q)=\inf \{r \geq 0: q \in[r, f(r))\}$. If this infimum is in fact a minimum then define $r_{q, i}=\ell(q)$ for all $i \geq 1$. Otherwise, choose any sequence $\left\{r_{q, i}\right\}_{i \geq 1}$ such that $r_{q, i} \searrow \ell(q)$ monotonously. Then we define $\mathbb{C}=\left\{r_{q, i}\right\}_{q \in \mathbb{Q} \cap[0, \infty), i \geq 1}$. Now let $x \in[0, \infty)$ be arbitrary. We want to prove that $x \in\left[r_{q, i}, f\left(r_{q, i}\right)\right)$ for some $q \in \mathbb{Q} \cap[0, \infty), i \geq 1$. First choose some $q \in[x, f(x)) \cap \mathbb{Q}$. By definition, it holds that $\ell(q) \leq x$. Now we distinguish two cases. If $\ell(q)=x$ then the infimum in the definition of $\ell(q)$ is in fact a minimum and we have $x \in\left[r_{q, 1}, f\left(r_{q, 1}\right)\right)$. On the other hand, if $\ell(q)<x$ then there exists a sufficiently large $i$ such that $r_{q, i}<x$. Since $x \leq q<f\left(r_{q, i}\right)$, we obtain $x \in\left[r_{q, i}, f\left(r_{q, i}\right)\right)$, thereby proving the lemma.

Theorem 2.2. If $\varepsilon_{c}(X, r)>0$ for all $r \geq 0$ then a.s. $X$ has no descending chain.

Proof. Consider the function $f:[0, \infty) \rightarrow[0, \infty)$ with $f(r)=r+\varepsilon(r)$, where $\varepsilon(r)=$ $\varepsilon_{c}(X, r) / 2$ if $\varepsilon_{c}(X, r)<\infty$, and $\varepsilon(r)=1$ otherwise (in fact, the only requirements on $\varepsilon(r)$ are $\varepsilon(r)>0$ and $\left.\varepsilon(r)<\varepsilon_{c}(X, r)\right)$. Clearly, this function fulfills the condition of Lemma 2.1. Furthermore, for each $\varphi=\left\{x_{n}\right\} \subset \mathbb{R}^{d}$ locally finite, let

$$
R(\varphi)= \begin{cases}\lim _{n \rightarrow \infty}\left|\tilde{x}_{n}-\tilde{x}_{n-1}\right| & \text { if } \varphi \text { contains a descending chain } \\ 0 & \text { otherwise, }\end{cases}
$$

where $\left\{\tilde{x}_{n}\right\} \subset \varphi$ denotes the descending chain of $\varphi$ whose index set is minimal in the lexicographic ordering. Using this notation, we obtain

$$
\begin{aligned}
& \mathbb{P}(X \text { has a descending chain }) \\
& \quad \leq \mathbb{P}\left(\bigcap_{\varepsilon>0}\{G(X, R, \varepsilon) \text { percolates }\}\right) \\
& \quad \leq \mathbb{P}\left(\bigcup_{r \in \mathbb{C} \cap[0, \infty)}\{R \in[r, r+\varepsilon(r)) \text { and } G(X, R, r+\varepsilon(r)-R) \text { percolates }\}\right) \\
& \quad \leq \sum_{r \in \mathbb{C} \cap[0, \infty)} \mathbb{P}(G(X, r, \varepsilon(r)) \text { percolates }) \\
& \quad=0
\end{aligned}
$$

where the second inequality follows from Lemma 2.1, and in the third inequality we used subadditivity of $\mathbb{P}$ and the relation $G(X, r, \varepsilon) \supset G(X, s, \varepsilon+r-s)$ for all $r \leq s<r+\varepsilon$.

\subsection{Examples}

We first provide some simple examples of point processes having positive annulus continuum percolation thresholds. In connection with this we consider point processes with a finite range of dependence.

Definition 2.3. Let $X \subset \mathbb{R}^{d}$ be an arbitrary point process. We say that $X$ has a finite range of dependence if there exists an integer $m>0$ such that, for any Borel sets $A, B \subset \mathbb{R}^{d}$ with $\rho(A, B)=\inf \{|x-y|: x \in A, y \in B\}>m$, it holds that the restrictions $\left.X\right|_{A}$ and $\left.X\right|_{B}$ of $X$ to the sets $A$ and $B$, respectively, are independent, where $\left.X\right|_{A}=X \cap A$ and $\left.X\right|_{B}=X \cap B$.

It is not difficult to see that the following point processes have a finite range of dependence: Cox processes whose random intensity field has a finite range of dependence, cluster processes whose primary process has a finite range of dependence and whose secondary process has a 
bounded support, and Matern hardcore processes whose primary point process has a finite range of dependence.

Now we can state the following result whose proof is postponed to Section 4.1, where it will be provided in a more general context.

Proposition 2.1. Let $X \subset \mathbb{R}^{d}$ be a stationary point process with finite range of dependence and locally finite second factorial moment measure that is absolutely continuous with respect to the Lebesgue measure in $\mathbb{R}^{2 d}$. Then $\varepsilon_{c}(X, r)>0$ holds for all $r \geq 0$.

By the results of Theorem 2.2 and Proposition 2.1, stationary point processes with a finite range of dependence and absolutely continuous second factorial moment measure do not possess descending chains. Therefore, by Theorem 2.1, the relative neighborhood graphs on such point processes are a.s. connected. Furthermore, there are some classes of stationary point processes for which conditions can be formulated that directly imply the almost-sure absence of descending chains; see [8]. In particular, the following results have been derived in Theorems 7.1-7.3 of [8], which we restate here for the convenience of the reader.

Proposition 2.2. Let $X$ be a Cox process with random intensity field $\left\{\xi_{x}\right\}_{x \in \mathbb{R}^{d}}$ which is stationary and satisfies

$$
\limsup _{k \rightarrow \infty} \frac{\sqrt[k]{\mathbb{E}\left(\xi_{0}^{k}\right)}}{k}<\infty
$$

Then a.s. $X$ has no descending chain.

Following [8] we write $\mathbb{N}$ for the family of all locally finite sets of $\mathbb{R}^{d}$ and $\mathcal{N}$ for the smallest $\sigma$-algebra of subsets of $\mathbb{N}$ such that the mappings $\varphi \rightarrow \varphi(B)$ are measurable for all Borel sets $B \subset \mathbb{R}^{d}$. For $\varphi \in \mathbb{N}$, denote by $\varphi^{(n)}$ the set of all $n$-tuples $\left(x_{1}, \ldots, x_{n}\right) \in \varphi^{n}$ with pairwise different entries. Note that $\varphi^{(n)}$ can be identified with the measure $\varphi^{(n)}: \mathscr{B}^{n d} \rightarrow[0, \infty]$ given by

$$
\varphi^{(n)}(B)=\sum_{\left(x_{1}, \ldots, x_{n}\right) \in \varphi^{(n)}} \mathbf{1}_{B}\left(x_{1}, \ldots, x_{n}\right), \quad B \in \mathcal{B}^{n d},
$$

where $\mathscr{B}^{n d}$ denotes the Borel $\sigma$-algebra of $\mathbb{R}^{n d}$ and $\mathbf{1}_{B}$ is the indicator of the set $B \in \mathcal{B}^{n d}$. Furthermore, we use the notation $m^{[n]}=m(m-1) \cdots(m-n+1)$ for any $1 \leq n \leq m$.

Proposition 2.3. Let $X$ be a stationary Poisson cluster process, and let $\Pi_{n}$ denote the conditional distribution of a single cluster centered at the origin, given that this cluster has exactly $n$ points. Assume that there exists an $M>0$ such that

$$
\iint \mathbf{1}_{B}\left(x_{2}-x_{1}, \ldots, x_{k+1}-x_{1}\right) \varphi^{(k+1)}\left(\mathrm{d}\left(x_{1}, \ldots, x_{k+1}\right)\right) \Pi_{n}(\mathrm{~d} \varphi) \leq M^{k} n^{[k+1]} v_{k d}(B),
$$

holds for all $n \geq 0, k \in\{0,1, \ldots, n-1\}$, and $B \in \mathcal{B}^{\text {nd }}$, where $v_{k d}$ denotes the Lebesgue measure on $\mathcal{B}^{k d}$. Furthermore, assume that the cluster size distribution $\left\{p_{k}, k \geq 0\right\}$ has a finite exponential moment, i.e. $\sum_{k=0}^{\infty} p_{k} z^{k}<\infty$ for some $z>1$. Then $X$ a.s. does not have descending chains.

Suppose that $X$ is a stationary Gibbs point process, i.e. there exists a measurable function $e: \mathbb{R}^{d} \times \mathbb{N} \rightarrow[0, \infty)$ (the conditional intensity) satisfying $e(x, \varphi)=0$ if $x \in \varphi$, and

$$
\mathbb{E}\left(\sum_{x \in X} f(x, X \backslash\{x\})\right)=\mathbb{E}\left(\int_{\mathbb{R}^{d}} e(x, X) f(x, X) \mathrm{d} x\right)
$$


for all measurable functions $f: \mathbb{R}^{d} \times \mathbb{N} \rightarrow[0, \infty)$. We furthermore assume that there exists a measurable function $\mu: \mathbb{N} \rightarrow[0, \infty)$ satisfying $e(x, \varphi)=\mu\left(T_{x} \varphi\right)$, where $T_{x}: \mathbb{N} \rightarrow \mathbb{N}$ denotes the shift operator given by $T_{x} \varphi=\varphi-x$. Using this notation, we can state the following result.

Proposition 2.4. Let $c>0$, and let $X$ be a Gibbs process as described above fulfilling

$$
\mathbb{E}\left(e\left(x_{1}, X\right) \cdots e\left(x_{n}, X \cup\left\{x_{1}, \ldots, x_{n-1}\right\}\right)\right)<c^{n} n !
$$

for all $n \geq 1$ and all pairwise distinct $x_{1}, \ldots, x_{n} \in \mathbb{R}^{d}$. Then a.s. $X$ has no descending chain.

\section{Connectivity of creek-crossing graphs}

\subsection{Generalized descending chains}

The sufficient condition for the connectivity of $G_{n}(X)$ to be derived uses a generalization of the notion of descending chains considered in Section 2 for $n=2$. We also specify a family of continuum percolation models such that the strict positivity of percolation thresholds will imply the almost-sure absence of generalized descending chains.

Definition 3.1. Let $b>0$, and let $\varphi \subset \mathbb{R}^{d}$ be a locally finite set. We say that $\varphi$ has a $b$-bounded generalized descending chain if there exists an infinite sequence $x_{1}, x_{2}, \ldots \in \varphi$ and an ordered set $I=\left\{i_{1}, i_{2}, \ldots\right\} \subset\{1,2, \ldots\}$ with the following properties:

1. $\left|i_{j+1}-i_{j}\right| \leq 2$ for all $j \geq 0$,

2. $0<\left|x_{i}-x_{i+1}\right| \leq b$ for all $i \geq 1$,

3. $\left|x_{i_{j}+1}-x_{i_{j}}\right|<\left|x_{i_{j-1}+1}-x_{i_{j-1}}\right|$ for all $j \geq 2$,

where we use the convention $i_{0}=0$. We say that $\varphi$ has a generalized descending chain if there exists some $b>0$ such that $\varphi$ has a $b$-bounded generalized descending chain; see Figure 1 .

The following auxiliary result is useful.

Lemma 3.1. Let $\varphi \subset \mathbb{R}^{d}$ be locally finite. Suppose that $\varphi$ contains a generalized descending chain. Then $\varphi$ contains a generalized descending chain consisting of pairwise distinct elements.

Proof. We show how to transform a given $b$-bounded generalized descending chain $x_{1}, x_{2}, \ldots$ into a $2 b$-bounded generalized descending chain consisting of pairwise distinct elements. The proof is based on Lawler's method of loop erasure [13]. Let us begin by proving that each element $x_{i}$ of the descending chain can be visited only finitely many times. Suppose to the contrary that there exists an infinite sequence $k_{1}<k_{2}<\cdots$ with $x_{k_{1}}=x_{k_{2}}=\cdots$. Then, by

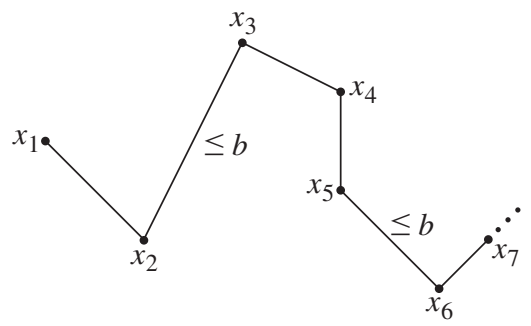

Figure 1: Generalized descending chain. 

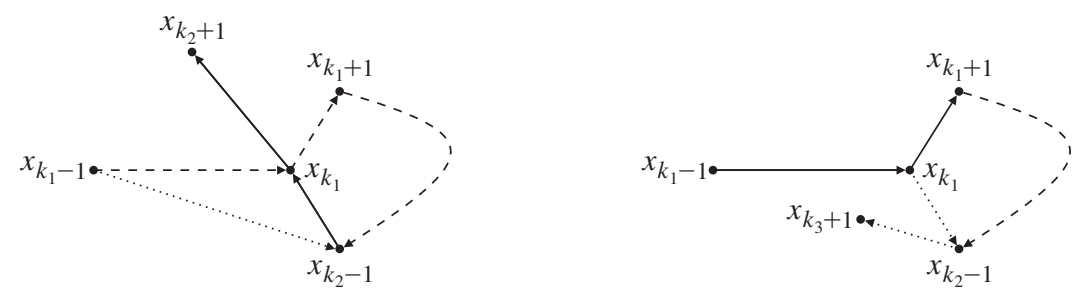

FIGURE 2: New paths with cancelled (dashed lines) and added (dotted lines) edges.

Definition 3.1, we have infinitely many points of $\varphi$ in the ball $B\left(x_{k_{1}}, b\right)$ centered at $x_{k_{1}}$ with radius $b$. This contradicts the local finiteness property of $\varphi$. Choose the smallest $k_{1}$ with the property that $x_{k_{1}}$ is visited at least twice. Furthermore, let $k_{2}$ be chosen as the last index such that $x_{k_{2}}=x_{k_{1}}$. First let us suppose that $k_{1}-1 \in I$ or that $k_{2} \in I$. In this case, we may simply remove the loop from $x_{k_{1}+1}$ and $x_{k_{2}}$ from the sequence $x_{1}, x_{2}, \ldots$ Now suppose that both $k_{1}-1 \notin I$ and $k_{2} \notin I$. Here, again, we need to distinguish two cases. First assume that $x_{k_{2}-1}$ is not visited again after the time $k_{2}$. Then delete all vertices from the loop between $k_{1}$ and $k_{2}$ except for the vertex $x_{k_{2}-1}$ and redefine $x_{k_{1}}=x_{k_{2}-1}$ (see the left-hand diagram of Figure 2). On the other hand, suppose that $x_{k_{2}-1}$ is visited again after time instant $k_{2}$. Let $k_{3}$ be the last such time. Then we define $x_{k_{1}+1}=x_{k_{2}-1}$ and continue the path at $x_{k_{3}+1}$ (see the right-hand diagram of Figure 2). These steps ensure that in the new chain $x_{k_{1}}$ is visited only once. Iterating this procedure yields the desired chain.

Now we can prove a criterion for deterministic locally finite sets $\varphi \subset \mathbb{R}^{d}$ ensuring the connectivity of $G_{n}(\varphi)$, which is an extension of the criterion derived in Theorem 2.1.

Theorem 3.1. Let $\varphi \subset \mathbb{R}^{d}$ be locally finite. If $\varphi$ has no generalized descending chains then $G_{n}(\varphi)$ is connected for all $n \geq 2$.

Proof. First observe that $G_{n}(\varphi)$ is always connected if $\varphi$ is finite. Suppose now that $\varphi$ is an infinite set such that $G_{n}(\varphi)$ is not connected. As in the proof of Theorem 2.1, we write $x \neq y$ if $x, y \in \varphi$ do not belong to the same connected component of $G_{n}(\varphi)$; otherwise, we write $x \sim y$. Then, an infinite sequence $x_{1}, x_{1}^{\prime}, x_{2}, x_{2}^{\prime}, \ldots \in \varphi$ forming a generalized descending chain can be constructed as follows. Choose an arbitrary $x_{1} \in \varphi$, and put

$$
x_{1}^{\prime}=\operatorname{argmin}_{y \in \varphi}\left(\left|x_{1}-y\right|: x_{1} \not \gamma\right) .
$$

In case the argmin is not unique, choose an arbitrary element of this set. Now define

$$
x_{1}^{\prime \prime}=\operatorname{argmin}_{y \in \varphi}\left(\left|x_{1}^{\prime}-y\right|: x_{1}^{\prime} \nsim y\right) .
$$

Since $x_{1}^{\prime} \nsucc x_{1}^{\prime \prime}$, there exists $z_{0}=x_{1}^{\prime}, z_{1}, \ldots, z_{\ell}=x_{1}^{\prime \prime} \in \varphi$ satisfying $\ell \leq n$ and $\max _{i}\left\{\mid z_{i}-\right.$ $\left.z_{i+1} \mid\right\}<\left|x_{1}^{\prime}-x_{1}^{\prime \prime}\right|$. Let $j=\min \left(i: z_{i} \nsim x_{1}^{\prime}\right)$. Now define $x_{2}=z_{j-1}$ and $x_{2}^{\prime}=z_{j}$. Then we have $x_{2}^{\prime} \nsucc x_{2}$ and, consequently, $\left|x_{1}-x_{1}^{\prime}\right| \geq\left|x_{1}^{\prime \prime}-x_{1}^{\prime}\right|>\left|x_{2}-x_{2}^{\prime}\right|$. Furthermore, suppose that, for some $k \geq 2$, a sequence $x_{1}, x_{1}^{\prime}, \ldots, x_{k}, x_{k}^{\prime} \in \varphi$ is given such that $x_{1} \nsucc x_{1}^{\prime}, x_{2} \nsucc x_{2}^{\prime}$, $\ldots, x_{k} \nsucc x_{k}^{\prime}, \max _{i}\left(\left|x_{i}^{\prime}-x_{i+1}\right|\right) \leq(n-1)\left|x_{1}-x_{1}^{\prime}\right|$, and $\left|x_{1}-x_{1}^{\prime}\right|>\cdots>\left|x_{k}-x_{k}^{\prime}\right|$. Put

$$
x_{k}^{\prime \prime}=\operatorname{argmin}_{y \in \varphi}\left(\left|x_{k}^{\prime}-y\right|: x_{k}^{\prime} \not y\right) .
$$

It is clear that $\left|x_{k}-x_{k}^{\prime}\right| \geq\left|x_{k}^{\prime}-x_{k}^{\prime \prime}\right|$. Since $x_{k}^{\prime} \nsucc x_{k}^{\prime \prime}$, there exists $z_{0}=x_{k}^{\prime}, z_{1}, \ldots, z_{\ell}=x_{k}^{\prime \prime} \in \varphi$ satisfying $\ell \leq n$ and $\max _{i}\left\{\left|z_{i}-z_{i+1}\right|\right\}<\left|x_{k}^{\prime}-x_{k}^{\prime \prime}\right|$. Let $j=\min \left(i: z_{i} \nsucc x_{k}^{\prime}\right)$. Now define 


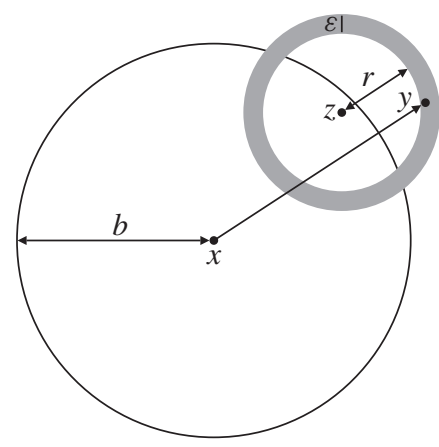

FIGURE 3: Connection rule in $G(\varphi, b, r, \varepsilon)$.

$x_{k+1}=z_{j-1}$ and $x_{k+1}^{\prime}=z_{j}$. Then we have $x_{k+1}^{\prime} \nsim x_{k+1}$ and, consequently,

$$
\left|x_{k}-x_{k}^{\prime}\right| \geq\left|x_{k}^{\prime \prime}-x_{k}^{\prime}\right|>\left|x_{k+1}-x_{k+1}^{\prime}\right|
$$

and

$$
(n-1)\left|x_{1}-x_{1}^{\prime}\right| \geq \sum_{i=0}^{\ell-2}\left|z_{i}-z_{i+1}\right| \geq\left|x_{k+1}-x_{k}^{\prime}\right|,
$$

which proves the theorem.

Corollary 3.1. Let $X \subset \mathbb{R}^{d}$ be a point process. If $X$ has no generalized descending chains with probability 1 then $G_{n}(X)$ is a.s. connected for all $n \geq 2$.

\subsection{Annulus continuum percolation threshold}

The following definition generalizes the notion of the annulus continuum percolation threshold introduced in Definition 2.2, which is obtained as a special case for $x=z$.

Definition 3.2. Let $\varphi \subset \mathbb{R}^{d}$ be a locally finite set, let $r, b \geq 0$, and let $\varepsilon>0$. The directed graph $G(\varphi, b, r, \varepsilon)$ with vertex set $\varphi$ is defined as follows. Assume that $x \in \varphi$ is connected by an oriented edge to $y \in \varphi$ if and only if there exists a vertex $z \in \varphi$ such that $|x-z| \leq b$ and $r<|z-y|<r+\varepsilon$; see Figure 3. Furthermore, for a point process $X \subset \mathbb{R}^{d}$, let $\varepsilon_{c}(X, b, r)=\inf \{\varepsilon>0: \mathbb{P}(G(X, b, r, \varepsilon)$ percolates $)>0\}$, where a (directed) graph is said to percolate if it contains an infinite self-avoiding directed path.

It is easy to see that $G(\varphi, b, r, \varepsilon)$ percolates for $r=\lim _{n \rightarrow \infty}\left|x_{i_{n}+1}-x_{i_{n}}\right|$ and all $\varepsilon>0$ if $x_{1}, x_{2}, \ldots$ is a $b$-bounded generalized descending chain of $\varphi$. Moreover, a probabilistic counterpart of this elementary statement is true.

Theorem 3.2. Let $b \geq 0$ be arbitrary but fixed. If $\varepsilon_{c}(X, b, r)>0$ for all $r \geq 0$ then a.s. $X$ has no b-bounded generalized descending chain.

The proof of Theorem 3.2 is analogous to that of Theorem 2.2, where it suffices to replace $\varepsilon_{c}(X, r)$ by $\varepsilon_{c}(X, b, r)$, the phrase descending chain by the $b$-bounded generalized descending chain, and $G(X, R, \varepsilon)$ by $G(X, b, R, \varepsilon)$.

\section{Examples}

We now consider two classes of stationary point processes that a.s. do not admit generalized descending chains. First we consider point processes with positive percolation thresholds. 
The second class consists of several examples for which one can use a certain moment condition to prove the a.s. absence of generalized descending chains.

\subsection{Point processes with positive percolation thresholds}

In this section we show that both point processes with a finite range of dependence as well as processes whose factorial moment measures satisfy some product-form domination condition have positive percolation thresholds.

4.1.1. Finite range of dependence. In order to extend the statement of Proposition 2.1, we apply an auxiliary result given in [14], see Lemma 4.1 below, where the following notion of stochastic dominance of probability measures is used.

Definition 4.1. Let $\mathbb{Z} \subset \mathbb{R}$ denote the set of all integers, and consider $\{0,1\}^{\mathbb{Z}^{d}}$ as a topological space endowed with the product topology. Let $\mu, v: \mathscr{B}\left(\{0,1\}^{\mathbb{Z}^{d}}\right) \rightarrow[0,1]$ be probability measures, where $\mathscr{B}\left(\{0,1\}^{\mathbb{Z}^{d}}\right)$ denotes the Borel $\sigma$-algebra over $\{0,1\}^{\mathbb{Z}^{d}}$. Then, $\mu$ is said to stochastically dominate $v$ if, for any continuous (componentwise) increasing function $f:\{0,1\}^{\mathbb{Z}^{d}} \rightarrow \mathbb{R}$, it holds that $\int_{\{0,1\}^{\mathbb{Z}^{d}}} f(\omega) \mu(\mathrm{d} \omega) \geq \int_{\{0,1\}^{\mathbb{Z}^{d}}} f(\omega) v(\mathrm{~d} \omega)$.

Definition 4.2. We denote by $\mathcal{C}(d, k, p)$ the class of $\mathbb{Z}^{d}$-indexed random fields $\left\{X_{s}\right\}_{s \in \mathbb{Z}^{d}}$ satisfying the conditions

1. $\left\{X_{s}\right\}_{s \in \mathbb{Z}^{d}}$ is $k$-dependent, i.e. for all $A, B \subset \mathbb{Z}^{d}$ with $d_{\infty}(A, B) \geq k$, the random fields $\left\{X_{s}\right\}_{s \in A}$ and $\left\{X_{t}\right\}_{t \in B}$ are independent, where

$$
d_{\infty}(A, B)=\inf _{a \in A, b \in B} \max _{i=1, \ldots, d}\left|a_{i}-b_{i}\right|,
$$

2. $\mathbb{P}\left(X_{s}=1\right) \geq p$ for all $s \in \mathbb{Z}^{d}$.

Lemma 4.1. Let $d, k \geq 1$. When $p$ is large enough, every random field in $\mathcal{C}(d, k, p)$ dominates a product random field with density $0<\rho<1$ (where $\rho$ is a constant depending on $d, k$, and $p$ ). One can make the density of these product random fields become arbitrarily close to 1 by taking p large enough.

Since $\varepsilon_{c}(X, r) \geq \varepsilon_{c}(X, b, r)$ for any $b, r \geq 0$, the following result is an extension of Proposition 2.1.

Proposition 4.1. Let $X \subset \mathbb{R}^{d}$ be a stationary point process with a finite range of dependence and locally finite second factorial moment measure that is absolutely continuous with respect to the Lebesgue measure in $\mathbb{R}^{2 d}$. Then $\varepsilon_{c}(X, b, r)>0$ holds for all $b, r \geq 0$.

Proof. Let $b, r \geq 0$ be arbitrary fixed numbers. The idea is to discretize $\mathbb{R}^{d}$ and then to apply Lemma 4.1. For $x \in \mathbb{Z}^{d}$ and $s>0$, define the box $Q_{s}(x)=x \oplus[-s / 2, s / 2]^{d}$ and the ball $B_{s}(x)=\left\{y \in \mathbb{R}^{d}:|x-y| \leq s\right\}$, where $A \oplus B=\{x+y: x \in A, y \in B\}$ denotes Minkowski addition of $A, B \subset \mathbb{R}^{d}$. Now let $0<\varepsilon<1$ be arbitrary (the value of $\varepsilon$ will be specified later). Consider the lattice $\mathcal{L}=(V, E)$ with $V=\mathbb{Z}^{d}$ and $E=\left\{\{x, y\}: x, y \in \mathbb{Z}^{d}\right.$, $x \neq y,\left|x_{i}-y_{i}\right| \leq 1$ for all $\left.1 \leq i \leq d\right\}$, where $x=\left(x_{1}, \ldots, x_{d}\right)$ and $y=\left(y_{1}, \ldots, y_{d}\right)$. Then we define a site percolation model $\left(Y_{z}\right)_{z \in \mathbb{Z}^{d}}$ on $\mathcal{L}$ as follows. We say that a site $v \in V$ is open-and write $Y_{v}=0$-if there exist $x, y \in X$ such that $x \in(r+b+1) Q_{1}(v)$ and there exists a (directed) edge from $x$ to $y$ in $G(X, b, r, \varepsilon)$ (note that we do not necessarily assume that $\left.y \in(r+b+1) Q_{1}(v)\right)$. Otherwise, the site $v \in V$ is said to be closed. First, it is clear that if $G(X, b, r, \varepsilon)$ percolates then the associated site model does so. Thus, it is sufficient to show that the site model a.s. does not percolate, when $\varepsilon$ is chosen small enough. 
First, for $0<\rho<1$, consider Bernoulli site percolation $\left(Y_{z}^{\prime}\right)_{z \in \mathbb{Z}^{d}}$ on the lattice $\mathcal{L}$ and where the probability for any fixed site $z \in \mathbb{Z}^{d}$ to be closed-write $Y_{z}^{\prime}=1$-is given by $\rho$. It is well known from classical percolation theory (see [10, Theorem 1.33]) that, by choosing $\rho$ sufficiently close to 1 , the set of open sites a.s. does not percolate. In particular, the proof of Proposition 4.1 is completed, if we can show that $\left(Y_{z}\right)_{z \in \mathbb{Z}^{d}}$ dominates $\left(Y_{z}^{\prime}\right)_{z \in \mathbb{Z}^{d}}$. By Lemma 4.1, it suffices to show that there exist $k \geq 1$ such that, for all $0<p<1$, we can find $\varepsilon>0$ with $\left(Y_{z}\right)_{z \in \mathbb{Z}^{d}} \in \mathcal{C}(d, k, p)$. First observe that, for any $0<\varepsilon<1$ and any $z \in \mathbb{Z}^{d}$, the event $Y_{z}=0$ depends only on $X \cap\left((r+b+1) v+3(r+b+1) Q_{1}(o)\right)$. Since the range of dependence of $X$ is finite, this implies that we can find some $k \geq 1$ such that $\left(Y_{z}\right)_{z \in \mathbb{Z}^{d}}$ is $k$-dependent. Finally, let us show that $\lim _{\varepsilon \rightarrow 0} \mathbb{P}\left(Y_{o}=1\right) \rightarrow 1$. Let $\rho^{(2)}\left(x_{1}, x_{2}\right)$ be the density of the second factorial moment measure of $X$, and observe that

$\mathbb{P}(v$ is open $)$

$$
\begin{aligned}
& \leq \mathbb{E}\left(\sum_{X_{i}, X_{j}, X_{i} \neq X_{j}} \mathbf{1}_{\left((r+b+1) Q_{1}(v)\right) \oplus\left(B_{b}(0)\right)}\left(X_{i}\right) \mathbf{1}_{\{(y, z): r<|y-z|<r+\varepsilon\}}\left(X_{i}, X_{j}\right)\right) \\
& =\lambda^{2} \int \rho^{(2)}\left(x_{1}, x_{2}\right) \mathbf{1}_{\left((r+b+1) Q_{1}(v)\right) \oplus\left(B_{b}(0)\right)}\left(x_{1}\right) \mathbf{1}_{\{(y, z): r<|y-z|<r+\varepsilon\}}\left(x_{1}, x_{2}\right) \mathrm{d} x_{1} \mathrm{~d} x_{2} .
\end{aligned}
$$

For $\varepsilon \rightarrow 0$, the integrand tends to 0 . Thus, it follows from an application of dominated convergence that $\mathbb{P}(v$ is open $)$ becomes arbitrarily small as $\varepsilon$ approaches 0 .

4.1.2. Sub-Poisson processes. Using ideas from [7], the positivity of the percolation thresholds can also be proven for so-called $\alpha$-weakly sub-Poisson processes. To give a formal proof of this statement, we need to introduce a further notion which can be seen as a discretized path-oriented version of the directed graph $G(\varphi, b, r, \varepsilon)$ introduced in Definition 3.2. For $0<\varepsilon<1, r>0$, and $x \in \mathbb{R}^{d}$, we use the notation $\mathbb{Z}_{\varepsilon}^{d}=\varepsilon \mathbb{Z}^{d}, Q_{\varepsilon}(x)=x \oplus[-\varepsilon / 2, \varepsilon / 2]^{d}$, and $R(r, \varepsilon)=\left\{y \in \mathbb{R}^{d}: r<|y|<r+\varepsilon\right\}$.

Definition 4.3. Let $r \geq 0,0<\varepsilon<1$, and $b \geq r+\varepsilon$, and let $\varphi \subset \mathbb{Z}_{\varepsilon}^{d}$ be arbitrary. We say that $\varphi$ has a $(b, r, \varepsilon)$-chain if there exists an infinite sequence $z_{1}, z_{2}, \ldots \in \varphi$ and an ordered set $I=\left\{i_{1}, i_{2}, \ldots\right\} \subset\{1,2, \ldots\}$ with the properties

1. $\left|i_{j+1}-i_{j}\right| \leq 2$ for all $j \geq 0$,

2. $Q_{\varepsilon}\left(z_{i+1}\right) \cap\left(Q_{\varepsilon}\left(z_{i}\right) \oplus B_{b}(0)\right) \neq \varnothing$ for all $i \geq 1$,

3. $Q_{\varepsilon}\left(z_{i_{j}+1}\right) \cap\left(Q_{\varepsilon}\left(z_{i_{j}}\right) \oplus R(r, \varepsilon)\right) \neq \varnothing$ for all $j \geq 1$,

where we use the convention $i_{0}=0$.

The following auxiliary result is similar to Lemma 3.1.

Lemma 4.2. Let $r \geq 0,0<\varepsilon<1$, and $b \geq r+\varepsilon$, and let $\varphi \subset \mathbb{Z}_{\varepsilon}^{d}$. Suppose that $\varphi$ contains $a$ $(b, r, \varepsilon)$-chain $z_{1}, z_{2}, \ldots$ with the additional property that every $z_{n}$ is only visited a finite number of times. Then $\varphi$ contains $a(2 b+\sqrt{d} \varepsilon, r, \varepsilon)$-chain consisting of pairwise distinct elements.

Proof. We show how to transform a given $(b, r, \varepsilon)$-chain $z_{1}, z_{2}, \ldots$ into a $(2 b+\sqrt{d} \varepsilon, r, \varepsilon)$ chain consisting of pairwise distinct elements. The proof is based on Lawler's method of loop erasure [13]. Choose the smallest $k_{1}$ with the property that $z_{k_{1}}$ is visited at least twice. 
Furthermore, let $k_{2}$ be chosen as the last index such that $z_{k_{2}}=z_{k_{1}}$. First let us suppose that $k_{1}-1 \in I$ or that $k_{2} \in I$. In this case, we may simply remove the loop from $z_{k_{1}+1}$ and $z_{k_{2}}$ from the sequence $z_{1}, z_{2}, \ldots$. Now suppose that both $k_{1}-1 \notin I$ and $k_{2} \notin I$. Here, again, we need to distinguish two cases. First, assume that $z_{k_{2}-1}$ is not visited again after the time $k_{2}$. Then delete all vertices from the loop between $k_{1}$ and $k_{2}$ except for the vertex $z_{k_{2}-1}$, and redefine $z_{k_{1}}=z_{k_{2}-1}$. Second, suppose that $z_{k_{2}-1}$ is visited again after time instant $k_{2}$. Let $k_{3}$ be the last such time. Then we define $z_{k_{1}+1}=z_{k_{2}-1}$ and continue the path at $z_{k_{3}+1}$. These steps ensure that in the new chain $z_{k_{1}}$ is visited only once. Iterating this procedure yields the desired chain.

Definition 4.4. Let $X$ be a point process whose $n$th factorial moment measure is absolutely continuous with respect to the $n d$-dimensional Lebesgue measure for all $n \geq 1$. If there exists $c>0$ such that, for all $n \geq 1$, the respective densities $\rho^{(n)}$ satisfy $\rho^{(n)}\left(x_{1}, \ldots, x_{n}\right) \leq c^{n}$ for all $\left(x_{1}, \ldots, x_{n}\right) \in \mathbb{R}^{n d}$, then $X$ is called $\alpha$-weakly sub-Poisson.

Examples of $\alpha$-weakly sub-Poisson processes can be found in, e.g. [7].

Proposition 4.2. Let $X$ be an $\alpha$-weakly sub-Poisson process. Then $\varepsilon_{c}(X, b, r)>0$ holds for all $r, b \geq 0$.

Proof. It suffices to prove that there exists $n \geq 1$ such that $X$ does not contain an infinite path in $G(X, b, r, 1 / n)$ starting from $Q_{1 / n}\left(z_{1}\right)$ for all $z_{1} \in \mathbb{Z}_{1 / n}$. Note also that every such infinite path induces a $(b, r, 1 / n)$-chain with the property that each element is only visited a finite number of times (this follows from the local finiteness property of $X$ ). Using Lemma 4.2, it thus suffices to prove the a.s. absence of $(b, r, 1 / n)$-chains starting at $z_{1}$ and consisting of pairwise distinct elements. Let $m \geq 0,0 \leq \ell \leq m$, and $I=\left\{i_{1}, \ldots, i_{\ell}\right\} \subset\{0, \ldots, m-1\}$. Then we define the set $A_{I, z_{1}, b, r, n} \subset \mathbb{R}^{d(m-1)}$ as $A_{I, z_{1}, b, r, n}=B \cap C \cap D \cap E$, where

$$
\begin{aligned}
& B=\left\{\left(z_{2}, \ldots, z_{m}\right): 0<i_{j}-i_{j-1} \leq 2 \text { for all } 1 \leq j \leq \ell-1\right\}, \\
& C=\left\{\left(z_{2}, \ldots, z_{m}\right): Q_{1 / n}\left(z_{i+1}\right) \cap\left(Q_{1 / n}\left(z_{i}\right) \oplus B_{b}(0)\right) \neq \varnothing \text { for all } 1 \leq i \leq m-1\right\}, \\
& D=\left\{\left(z_{2}, \ldots, z_{m}\right): Q_{1 / n}\left(z_{i_{j}+1}\right) \cap\left(Q_{1 / n}\left(z_{i_{j}}\right) \oplus R\left(r, \frac{1}{n}\right)\right) \neq \varnothing \text { for all } 1 \leq j \leq \ell-1\right\}, \\
& E=\left\{\left(z_{2}, \ldots, z_{m}\right): z_{i} \neq z_{j} \text { for all } i \neq j\right\} .
\end{aligned}
$$

Thus, we may think of $A_{I, z_{1}, b, r, n}$ as a set of $(b, r, n)$-chains of length $m$ where the set $I$ is already specified. Furthermore, it is easy to see that there are constants $C_{1}, C_{2}>1$ (only depending on $d$ ) satisfying

$$
\left|\left\{z \in \mathbb{Z}_{1 / n}^{d}: Q_{1 / n}(z) \cap\left(Q_{1 / n}(0) \oplus B_{b}(0)\right) \neq \varnothing\right\}\right| \leq C_{1} b^{d} n^{d}
$$

and

$$
\left|\left\{z \in \mathbb{Z}_{1 / n}^{d}: Q_{1 / n}(z) \cap\left(Q_{1 / n}(0) \oplus R\left(r, \frac{1}{n}\right)\right) \neq \varnothing\right\}\right| \leq C_{2} r^{d-1} n^{d-1} .
$$

From this we conclude that

$$
\left|A_{I, z_{1}, b, r, n}\right| \leq\left(C_{1} b^{d} n^{d}\right)^{m-1-|I|}\left(C_{2} r^{d-1} n^{d-1}\right)^{|I|} .
$$

Therefore, if $Y$ is an $\alpha$-weakly sub-Poisson point process, we obtain

$$
\begin{aligned}
& \mathbb{P}\left(\text { there is an infinite path in } G\left(X, b, r, \frac{1}{n}\right) \text { starting from } Q_{1 / n}\left(z_{1}\right)\right) \\
& \quad \leq \mathbb{P}\left(\bigcup_{I \subset\{1, \ldots, m\}}\left\{\left|X \cap Q_{1 / n}\left(z_{i}\right)\right| \geq 1\right\}\right)
\end{aligned}
$$




$$
\begin{aligned}
& \leq \sum_{I \subset\{1, \ldots, m\}} \sum_{\substack{\left(z_{2}, \ldots, z_{m}\right) \in A_{I, z 1}, b, r, n\\
}} \mathbb{E}\left(\left|X \cap Q_{1 / n}\left(z_{1}\right)\right| \cdots\left|X \cap Q_{1 / n}\left(z_{m}\right)\right|\right) \\
& \leq \sum_{\substack{I \subset\{1, \ldots, m\} \\
|I| \geq m / 2}}\left(C_{1} b^{d} n^{d}\right)^{m-1-|I|}\left(C_{2} r^{d-1} n^{d-1}\right)^{|I|}\left(c\left(\frac{1}{n}\right)^{d}\right)^{m} \\
& \leq C_{1}^{m} C_{2}^{m} b^{d m} c^{m} r^{(d-1) m} \sum_{\substack{I \subset\{1, \ldots, m\} \\
|I| \geq m / 2}} n^{-|I|} \\
& \leq\left(C_{1} C_{2} b^{d} c r^{(d-1)} 2 n^{-1 / 2}\right)^{m}
\end{aligned}
$$

for all $m \geq 1$, which, for sufficiently large $n$, tends to 0 as $m \rightarrow \infty$.

\subsection{Moment condition for the absence of generalized descending chains}

We now show that by strengthening the assumptions used in [8, Theorem 4.1], we can obtain another criterion for the absence of generalized descending chains. To make this precise, we need to recall some definitions and assumptions given in [8, Section 4].

Let $b \geq 1, x_{0} \in \mathbb{R}^{d}, 0 \leq a \leq u \leq b, n \geq \underset{\widetilde{A}}{ }$, and $I=\left\{i_{1}, \ldots, i_{m}\right\} \subset\{0, \ldots, n-1\}$. Then we define $\widetilde{A}_{I, x_{0}, a, u, b} \subset \mathbb{R}^{d n}$ as $\widetilde{A}_{I, x_{0}, a, u, b}=\widetilde{B} \cap \widetilde{C} \cap \widetilde{D}$, where

$$
\begin{aligned}
& \widetilde{B}=\left\{\left(x_{1}, \ldots, x_{n}\right): 0<i_{j}-i_{j-1} \leq 2 \text { for all } 1 \leq j \leq m\right\}, \\
& \widetilde{C}=\left\{\left(x_{1}, \ldots, x_{n}\right): u>\left|x_{i_{1}}-x_{i_{1}+1}\right|>\cdots>\left|x_{i_{m}}-x_{i_{m}+1}\right| \geq a\right\},
\end{aligned}
$$

and

$$
\widetilde{D}=\left\{\left(x_{1}, \ldots, x_{n}\right):\left|x_{i}-x_{i+1}\right| \leq b \text { for all } 0 \leq i \leq n-1\right\}
$$

(using the convention $i_{0}=-1$ ). Let $K>1$ be chosen such that $K>\kappa_{d}$ holds for all $d \geq 1$, where $\kappa_{d}$ is the volume of the $d$-dimensional unit ball. We first state two auxiliary results which will be useful for later computations.

Lemma 4.3. For all $I=\left\{i_{1}, \ldots, i_{m}\right\} \subset\{0, \ldots, n-1\}, n \geq 1, b \geq 1, x_{0} \in \mathbb{R}^{d}$, and all $b \geq u>a \geq 0$ satisfying $u^{d}-a^{d} \leq 1$, it holds that

$$
v_{d n}\left(\widetilde{A}_{I, x_{0}, a, u, b} \leq b^{d n} K^{n} \frac{\left(u^{d}-a^{d}\right)^{\lfloor n / 2\rfloor}}{(\lfloor n / 2\rfloor) !} .\right.
$$

Proof. The assertion is shown by induction on $n$. The case $n=1$ is clear. To prove the general case, let us first assume that $i_{1}=0$, where we write $\widetilde{I}=\left\{i_{2}-1, \ldots, i_{m}-1\right\}$. Then we have

$$
\begin{aligned}
v_{d n}\left(\widetilde{A}_{I, x_{0}, a, u, b}\right) & \leq \int \mathbf{1}_{\{(y, z): u>|y-z| \geq a\}}\left(x_{1}, x_{0}\right) v_{d(n-1)}\left(\widetilde{A}_{\left.\widetilde{I}_{, x_{1}, a,\left|x_{1}-x_{0}\right|, b}\right) \mathrm{d} x_{1}}\right. \\
& \leq \int \mathbf{1}_{\left\{\left(y, x_{0}\right): u>\left|y-x_{0}\right| \geq a\right\}}\left(x_{1}\right) \frac{b^{d(n-1)} K^{n-1}\left(\left|x_{1}-x_{0}\right|^{d}-a^{d}\right)^{(\lfloor(n-1) / 2\rfloor)}}{(\lfloor(n-1) / 2\rfloor) !} \mathrm{d} x_{1} \\
& =b^{d(n-1)} K^{n} \frac{\left(u^{d}-a^{d}\right)^{(\lfloor(n-1) / 2\rfloor)+1}}{((\lfloor(n-1) / 2\rfloor)+1) !} .
\end{aligned}
$$


Now suppose that $i_{1}=1$. Here we write $\tilde{I}=\left\{i_{2}-2, i_{3}-2, \ldots, i_{m}-2\right\}$ and compute

$$
\begin{aligned}
v_{d n}\left(\widetilde{A}_{I, x_{0}, a, u, b} \leq \leq\right. & \int \mathbf{1}_{\{(y, z): b \geq|y-z|\}}\left(x_{1}, x_{0}\right) \int \mathbf{1}_{\{(y, z): u>|y-z| \geq a\}}\left(x_{1}, x_{2}\right) \\
& \times v_{d(n-2)}\left(\widetilde{A}_{\tilde{I}, x_{2}, a,\left|x_{1}-x_{2}\right|, b}\right) \mathrm{d} x_{2} \mathrm{~d} x_{1} \\
\leq & \int \mathbf{1}_{\{(y, z): b \geq|y-z|\}}\left(x_{1}, x_{0}\right) \int \mathbf{1}_{\{(y, z): u>|y-z| \geq a\}}\left(x_{1}, x_{2}\right) \\
& \times b^{d(n-2)} K^{n-2} \frac{\left(\left|x_{1}-x_{2}\right|^{d}-a^{d}\right)^{\lfloor n / 2\rfloor-1}}{(\lfloor n / 2\rfloor-1) !} \mathrm{d} x_{2} \mathrm{~d} x_{1} \\
= & b^{d(n-1)} K^{n} \frac{\left(u^{d}-a^{d}\right)^{\lfloor n / 2\rfloor}}{(\lfloor n / 2\rfloor) !} .
\end{aligned}
$$

Let $X$ be a stationary point process with intensity $\lambda$. Furthermore, let $\left\{\xi_{x}\right\}_{x \in \mathbb{R}^{d}}$ denote a stationary random field with values in a shift-invariant subset $W \subset E^{\mathbb{R}^{d}}$ (where $(E, \mathcal{E}$ ) is any measurable space), and let $\mathcal{W}$ denote the $\sigma$-algebra generated by the mappings $\operatorname{ev}_{x}: \xi \mapsto \xi_{x}$. We also assume joint stationarity of $(X, \xi)$ and that there exists a function $\mu: \mathbb{N} \times W \rightarrow \mathbb{R}$ satisfying

$$
\lambda \mathbb{P}_{0}^{X, \xi}((X \backslash\{0\}, \xi) \in(\cdot, \cdot))=\mathbb{E}\left(\mu(X, \xi) \mathbf{1}_{(\cdot, \cdot)}(X, \xi)\right),
$$

where the Palm distribution $\mathbb{P}_{0}^{X, \xi}: \mathcal{N} \otimes \mathcal{W} \rightarrow[0,1]$ is defined by

$$
\mathbb{P}_{0}^{X, \xi}(A)=\lambda^{-1} \mathbb{E}\left(\sum_{X_{n} \in[0,1]^{d}} \mathbf{1}_{A}\left(T_{x}(X, \xi)\right)\right), \quad A \in \mathcal{N} \otimes \mathcal{W}
$$

Finally, writing

$$
e_{n}\left(x_{1}, \ldots, x_{n}\right)=\prod_{i=1}^{n} \mu\left(T_{x_{i}}\left(X \cup\left\{x_{1}, \ldots, x_{i-1}\right\}\right), T_{x_{i}} \xi\right),
$$

we assume that there exist $c_{n}>0$ satisfying

$$
\mathbb{E}\left(e_{n}\left(x_{1}, \ldots, x_{n}\right)\right) \leq c_{n} \text { and } \limsup _{n \rightarrow \infty} \frac{\sqrt[\lfloor n / 2\rfloor]{c_{n}}}{n}<\infty .
$$

The following auxiliary result is also useful. For $b \geq u>a \geq 0, x_{0} \in \mathbb{R}^{d}$, and $I \subset$ $\{0, \ldots, n-1\}$, we define

$$
\begin{aligned}
C_{n, I}\left(x_{0}, a, u, b\right)= & \left\{\varphi \in \mathbb{N}: \text { there exist } x_{1}, \ldots, x_{n} \in \varphi \text { with }\left(x_{1}, \ldots, x_{n}\right) \in \widetilde{A}_{I, x_{0}, a, u, b}\right. \\
& \text { and } \left.x_{i} \neq x_{j} \text { for } i \neq j\right\} .
\end{aligned}
$$

Furthermore, we use the abbreviating notation $\alpha_{k}(I)=I \cap[0 ; k], \beta_{k}(I)=(I-k) \cap[0 ; n-k]$, and $\gamma(k, I)=\sup \{\ell<k: \ell \in I\}$, with the convention that $\left|x_{-\infty}\right|=u$.

Lemma 4.4. It holds that

$$
\begin{aligned}
\mathbb{P}\left(X \in C_{n, I}(o, a, u, b)\right) \leq \int & \mathbf{1}_{\tilde{A}_{\alpha_{k}(I), 0, a, u, b}}\left(x_{1}, \ldots, x_{1}+\cdots+x_{k}\right) \\
& \times \mathbb{E}\left(e_{k}\left(0,-x_{k},-x_{k}-x_{k-1}, \ldots,-x_{k}-\cdots-x_{2}\right)\right. \\
& \left.\times \mathbf{1}_{C_{n-k, \beta_{k}(I)}\left(o, a,\left|x_{\gamma(k, I)+1}\right|, b\right)}(X)\right) \mathrm{d} x_{1} \cdots \mathrm{d} x_{k} .
\end{aligned}
$$


Proof. The assertion is shown by induction on $k$, where the case $k=0$ is trivial. Assume now that the claim is true for $0<k<n$. Then

$$
\begin{aligned}
& \mathbb{P}\left(X \in C_{n, I}(o, a, u, b)\right) \\
& \leq \int \mathbf{1}_{\widetilde{A}_{\alpha_{k}(I), o, a, u, b}}\left(x_{1}, x_{1}+x_{2}, \ldots, x_{1}+\cdots+x_{k}\right) \\
& \times \mathbb{E}\left(e_{k}\left(0,-x_{k}, \ldots,-x_{k}-\cdots-x_{2}\right) \mathbf{1}_{C_{n-k, \beta_{k}(I)}\left(o, a,\left|x_{\gamma(k, I)+1}\right|, b\right)}(X)\right) \mathrm{d} x_{1} \cdots \mathrm{d} x_{k} \\
& \leq \int \mathbf{1}_{\widetilde{A}_{\alpha_{k}(I), o, a, u, b}}\left(x_{1}, x_{1}+x_{2}, \ldots, x_{1}+\cdots+x_{k}\right) \\
& \times \mathbb{E}\left[\left(\mathbf{1}_{\beta_{k}(I)}(0) \mathbf{1}_{\{(y, z):|y|>|z| \geq a\}}\left(x_{\gamma(k, I)+1}, x_{k+1}\right)\right.\right. \\
& \left.+\mathbf{1}_{\{0, \ldots, n-k\} \backslash \beta_{k}(I)}(0) \mathbf{1}_{\{y: b \geq|y|\}}\left(x_{k+1}\right)\right) e_{k}\left(0,-x_{k}, \ldots,-x_{k}-\cdots-x_{2}\right) \\
& \left.\times \mathbf{1}_{C_{n-(k+1), \beta_{k+1}(I)}\left(o, a,\left|x_{\gamma(k+1, I)+1}\right|, b\right)}\left(T_{x_{k+1}} X \backslash\{0\}\right) X\left(\mathrm{~d} x_{k+1}\right)\right] \mathrm{d} x_{1} \cdots \mathrm{d} x_{k} \\
& \leq \int \mathbf{1}_{\widetilde{A}_{\alpha_{k+1}(I), o, a, u, b}}\left(x_{1}, x_{1}+x_{2}, \ldots, x_{1}+\cdots+x_{k+1}\right) \\
& \times \mathbb{E}\left(e_{k+1}\left(0,-x_{k+1},-x_{k+1}-x_{k}, \ldots,-x_{k+1}-\cdots-x_{2}\right)\right. \\
& \left.\times \mathbf{1}_{C_{n-(k+1), \beta_{k+1}(I)}\left(o, a,\left|x_{\gamma(k+1, I)+1}\right|, b\right)}(X)\right) \mathrm{d} x_{1} \cdots \mathrm{d} x_{k} \mathrm{~d} x_{k+1} \text {. }
\end{aligned}
$$

Now we are in a position to prove the main result of this section.

Proposition 4.3. Let $X$ be a stationary point process satisfying conditions (4.1) and (4.2). Then a.s. $X$ has no generalized descending chain.

Proof. By subadditivity, it suffices to prove that, for every $b \in \mathbb{Q} \cap[1, \infty)$, a.s., $X$ does not contain any $b$-bounded generalized descending chains. Furthermore, we write $C_{n}\left(x_{0}, a, u, b\right)=$ $\bigcup_{I \subset\{0, \ldots, n-1\}} C_{n, I}\left(x_{0}, a, u, b\right)$ and $C\left(x_{0}, a, u, b\right)=\bigcap_{n>1} C_{n}\left(x_{0}, a, u, b\right)$. Now suppose we could show that, for any $b \geq 1$ and any $u \geq a \geq 0$, there exists $\varepsilon_{b}(a)>0$ satisfying

$\mathbb{P}(X \in C(o, a, u, b))=0 \quad$ for all $b \geq u>a$ with $u-a<\varepsilon_{b}(a)$ and $u^{d}-a^{d}<1$.

Then choose a countable $\Lambda \subset[0, \infty)$ such that $[0, \infty)=\bigcup_{a \in \Lambda}\left[a, a+\varepsilon_{b}(a) / 2\right)$ - the existence of $\Lambda$ follows from Lemma 2.1. From subadditivity and stationarity, we then also deduce that $\mathbb{P}\left(\bigcup_{x \in \mathbb{Q}^{d}} \bigcup_{a \in \Lambda} C\left(x, a, a+\varepsilon_{b}(a) / 2, b\right)\right)=0$. But, if $\varphi$ has $b$-bounded generalized descending chains then it is easy to see that $\varphi \in \bigcup_{x \in \mathbb{Q}^{d}} \bigcup_{a \in \Lambda} C\left(x, a, a+\varepsilon_{b}(a) / 2, b\right)$. In particular, we obtain from, Lemma 4.4,

$$
\mathbb{P}\left(X \in C_{n, I}(a, u, b)\right) \leq\left(\int \tilde{A}_{I, o, a, u, b}\left(x_{1}, \ldots, x_{1}+\cdots+x_{n}\right) \mathrm{d} x_{1} \cdots \mathrm{d} x_{n}\right) c_{n}
$$

Using Lemma 4.3 and the existence of $c>0$ satisfying $c_{n} \leq c^{\lfloor n / 2\rfloor}(\lfloor n / 2\rfloor)$ ! for sufficiently large $n$ (use (4.2) and Stirling's formula), we obtain

$$
\mathbb{P}\left(X \in C_{n, I}(a, u, b)\right) \leq K^{n} b^{d n} \frac{\left(u^{d}-a^{d}\right)^{\lfloor n / 2\rfloor}}{\lfloor n / 2\rfloor !} c_{n} \leq K^{n} b^{d n}\left(u^{d}-a^{d}\right)^{\lfloor n / 2\rfloor} c^{\lfloor n / 2\rfloor},
$$

which converges to 0 for $n \rightarrow \infty$, provided that $u^{d}-a^{d}$ is sufficiently small. 
Note that conditions (4.1) and (4.2) are satisfied for Cox processes and Gibbs processes (see Corollaries 4.1 and 4.2 below). We also remark that in general Poisson cluster processes do not satisfy (4.1). But, on the other hand, there are many Poisson cluster processes which satisfy the conditions of Proposition 4.1.

Corollary 4.1. Let $X$ be a Cox process with stationary random intensity field $\left\{\xi_{x}\right\}_{x \in \mathbb{R}^{d}}$ satisfying

$$
\lim \sup \frac{\sqrt[\lfloor n / 2\rfloor]{\mathbb{E}\left(\xi_{0}^{n}\right)}}{n}<\infty .
$$

Then a.s. X has no generalized descending chains.

Proof. Condition (4.1) is satisfied for $\mu(\varphi, w)=w_{0}$. Repeated application of Hölder's inequality yields $\mathbb{E}\left(e_{n}\left(x_{1}, \ldots, x_{n}\right)\right)=\mathbb{E}\left(\prod_{i=1}^{n} \xi_{-x_{i}}\right) \leq \mathbb{E}\left(\xi_{0}^{n}\right)$. Thus, the assumption of the corollary implies (4.2).

Furthermore, [8, Theorem 7.3] may be easily adapted to the case of generalized descending chains. Assume that $X$ is a Gibbs process. Then, by definition, there exists a measurable function $e: \mathbb{R}^{d} \times \mathbb{N} \rightarrow[0, \infty)$ satisfying

$$
\mathbb{E}\left(\sum_{x \in X} f(x, X \backslash\{x\})\right)=\mathbb{E}\left(\int e(x, X) f(x, X) \mathrm{d} x\right)
$$

for all measurable functions $f: \mathbb{R}^{d} \times \mathbb{N} \rightarrow[0, \infty)$. We also assume that there exists a measurable function $\mu: \mathbb{N} \rightarrow[0, \infty)$ satisfying $e(x, X)=\mu\left(T_{X} X\right)$. Using this notation, we obtain the following result.

Corollary 4.2. Let $c>0$, and let $X$ be a stationary Gibbs process as described above. Moreover, assume that

$$
\mathbb{E}\left(e\left(x_{1}, X\right) \cdots e\left(x_{n}, X \cup\left\{x_{1}, \ldots, x_{n-1}\right\}\right)\right)<c^{n}\left(\left\lfloor\frac{n}{2}\right\rfloor\right) !
$$

holds for any $n \geq 1$ and $x_{1}, \ldots, x_{n} \in \mathbb{R}^{d}$. Then a.s. $X$ has no generalized descending chains.

Proof. Condition (4.1) is satisfied for $\mu(\varphi, w)=\mu(\varphi)$. Since we have $e_{n}\left(x_{1}, \ldots, x_{n}\right)=$ $e\left(x_{1}, X\right) \cdots e\left(x_{n}, X \cup\left\{x_{1}, \ldots, x_{n-1}\right\}\right)$, the required inequality (4.2) follows from the assumption of the corollary.

Note that in Corollaries 4.1 and 4.2 we impose stronger moment conditions than in Propositions 2.2 and 2.4 , respectively.

\section{An application to microstructure analysis of advanced materials}

Gaiselmann et al. [9] proposed a stochastic model which describes the three-dimensional morphology of nanoparticle systems in porous materials used for electrodes of high-temperature fuel cells. As the nanoparticles form the solid phase of these porous materials, they should be completely connected to each other. Therefore, a variant of the creek-crossing graph has been considered in [9] to model the nanoparticle systems. We briefly sketch the definition of this model and show how the results of the present paper can be used to prove the desired connectivity. The basic difference of the creek-crossing graph considered in [9] is a modification of the Euclidean distance. This can be made precise by the following definition. 
Definition 5.1. Let $V$ be an arbitrary set, and denote by $K_{V}=(V, E)$ the complete graph on $V$. Furthermore, let $\pi: E \rightarrow[0, \infty)$ be an arbitrary (symmetric) weight function. Then the creek-crossing graph $G_{n}(V, \pi)$ is a graph on the vertex set $V$, where, for any $x, y \in V$, an edge is drawn between $x$ and $y$ if and only if there does not exist an integer $m \leq n$ and a finite sequence of vertices $x_{0}=x, \ldots, x_{m}=y, x_{i} \in V$ for all $i=0, \ldots, m$ satisfying $\pi\left(\left\{x_{i}, x_{i+1}\right\}\right)<\pi(\{x, y\})$ for all $i=0, \ldots, m-1$.

It is not difficult to see that Definitions 3.1 and 3.2 can easily be extended to the case of an arbitrary weight function. Thus, it still makes sense to speak about $b$-bounded generalized descending chains and the graphs $G(V, \pi, b, r, \varepsilon)$. On the other hand, the proof of Theorem 3.1 makes use of the triangle inequality, so we cannot expect it to hold in the more general setting of the present section, without any additional conditions. Therefore, from now we additionally assume that $V=\varphi \subset \mathbb{R}^{d}$ is locally finite and that $\pi(\{x, y\})=|x-y| \cdot f(x, y)$, where $f: \mathbb{R}^{2} \rightarrow[0, \infty)$ is a certain function such that, for some constants $c_{1}, c_{2}>0$, it holds that $c_{1} \leq f(x, y) \leq c_{2}$ for all $x, y \in \mathbb{R}^{d}$.

Suppose that $V$ and $\pi$ are of this form. Then the proof of Theorem 3.1 can be easily adapted, even if the triangle inequality does not hold for $\pi$. Furthermore, the proof of Proposition 4.1 can also be transferred to the more general setting. This yields a large class of stationary point processes $X$ with the property that also the more general graphs $G_{n}(X, \pi)$ are a.s. connected.

\section{Acknowledgements}

The authors would like to thank the anonymous referee for careful reading of the manuscript. The referee's suggestions helped to substantially improve the quality of this paper. The authors would also like to thank G. Gaiselmann and M. Neumann for interesting comments. This work has been supported by Orange Labs through research grant number 46146063-9241. Christian Hirsch has been supported by a research grant from DFG Research Training Group 1100 at Ulm University.

\section{References}

[1] Aldous, D. J. (2009). Which connected spatial networks on random points have linear route-lengths? Preprint. Available at http://arxiv.org/abs/0911.5296v1.

[2] Aldous, D. And Lyons, R. (2007). Processes on unimodular random networks. Electron. J. Prob. 12, 14541508.

[3] Aldous, D. J. And Shun, J. (2010). Connected spatial networks over random points and a route-length statistic. Statist. Sci. 25, 275-288.

[4] Aldous, D. And Steele, J. M. (1992). Asymptotics for Euclidean minimal spanning trees on random points. Prob. Theory Relat. Fields 92, 247-258.

[5] Aldous, D. And Steele, J. M. (2004). The objective method: probabilistic combinatorial optimization and local weak convergence. In Probability on Discrete Structures (Encyclopedia Math. Sci. 110), ed. H. Kesten, Springer, Berlin.

[6] Alexander, K. S. (1995). Percolation and minimal spanning forests in infinite graphs. Ann. Prob. 23, 87-104.

[7] BŁaszczyszyn, B. and Yogeshwaran, D. (2014). On comparison of clustering properties of point processes. To appear in Adv. Appl. Prob.

[8] Daley, D. J. ANd Last, G. (2005). Descending chains, the lilypond model, and mutual-nearest-neighbour matching. Adv. Appl. Prob. 37, 604-628.

[9] Gaiselmann, G. et al. (2013). Stochastic 3D modeling of $\mathrm{La}_{0.6} \mathrm{Sr}_{0.4} \mathrm{CoO}_{3}-\delta$ cathodes based on structural segmentation of FIB-SEM images. Computational Materials Sci. 67, 48-62.

[10] Grimmett, G. (1999). Percolation, 2nd edn. Springer, Berlin.

[11] Holroyd, A. E. And Peres, Y. (2003). Trees and matchings from point processes. Electron. Commun. Prob. 8, 17-27.

[12] Last, G. (2006). Stationary partitions and Palm probabilities. Adv. Appl. Prob. 38, 602-620.

[13] LaWler, G. F. (1980). A self-avoiding random walk. Duke Math. J. 47, 655-693. 
[14] Liggett, T. M., Schonmann, R. H. and Stacey, A. M. (1997). Domination by product measures. Ann. Prob. 25, 71-95.

[15] Lyons, R., Peres, Y. and Schramm, O. (2006). Minimal spanning forests. Ann. Prob. 34, 1665-1692.

[16] NeuhäUser, D., Hirsch, C., Gloaguen, C. And Schmidt, V. (2012). On the distribution of typical shortest-path lengths in connected random geometric graphs. Queueing Systems 71, 199-220.

[17] Penrose, M. D. And Yukich, J. E. (2001). Central limit theorems for some graphs in computational geometry. Ann. Appl. Prob. 11, 1005-1041.

[18] Penrose, M. D. And Yukich, J. E. (2003). Weak laws of large numbers in geometric probability. Ann. Appl. Prob. 13, 277-303.

[19] Timár, Á. (2006). Ends in free minimal spanning forests. Ann. Prob. 34, 865-869.

[20] Toussaint, G. T. (1980). The relative neighbourhood graph of a finite planar set. Pattern Recognition 12, 261-268. 